\title{
Evaluation of mammography detected breast arterial calcifications as a predictor of coronary cardiac risk
}

\author{
Engy A. Ali ${ }^{1,2^{*}}$ D, Heba Fouad ${ }^{2}$, Naglaa A. Razek ${ }^{2}$, Ola M. Saeed ${ }^{2}$ and Sameh M. Helmy ${ }^{3}$
}

\begin{abstract}
Background: Cardiovascular disease is one of the leading causes of death so detection of breast arterial calcifications on annually screening mammography can predict the possibility of future cardiovascular problems.

Results: The 100 female patients were divided into two groups according to age: 1st group with age ranging from 40 to 60 years and 2 nd group with age ranging from 61 to 80 years. There is increased percentage of cardiac cases among the BAC-positive patients in the 2 nd group with a significant $p$ value $=0.022$ and this proved that there was a correlation between presence of BAC and being a cardiac case in the 2nd group, unlike the 1st group which showed no correlation.

Conclusion: Incidental detection of breast arterial calcification in mammography in females above 60 years warrants further evaluation of their coronary atherosclerotic state and risk of future development of serious coronary artery disease.
\end{abstract}

Keywords: Mammography, Breast arterial calcifications, Coronary artery disease

\section{Background}

Cardiovascular disease was one of the leading causes of mortality and morbidity in the world. One-third of deaths were due to cardiovascular disease, causing health care costs and loss of productivity. Cardiovascular disease was the result of systemic arterial disease. Arterial calcification was considered a marker of overall atherosclerotic disease and therefore was a marker for future cardiovascular events. Numerous studies had been done to estimate coronary artery calcification noninvasively by CT. The recent meta-analysis with asymptomatic patients showed moderate increased risk (relative risk 2.1; 95\% confidence interval [CI], 1. 6-2.9) for cardiac events. Aortic calcification identified at chest radiography tends to be independently related to coronary heart disease (CHD), with an increased risk of 1.1 to 1.3.

\footnotetext{
* Correspondence: drengyali78@gmail.com

${ }^{1}$ Radiology Department (Women's Imaging Unit), Kasr El-Ainy Hospital ,Cairo University, Cairo, Egypt

${ }^{2}$ Diagnostic and Intervention Radiology Department, Cairo University

Hospitals, Kasr Al-Ainy, El-Manial, Cairo 11956, Egypt

Full list of author information is available at the end of the article
}

Vascular calcification other than in coronary arteries has also been investigated [1].

Vascular calcifications which were seen via mammography were called Mönckeberg calcifications, and involve the middle layer of the arteries. These calcifications were totally diffuse and thin, and tend to take the entire circumference of the peripheral arteries, resulting in rigid vessels with little capacity for distension [2]. Calcifications in patients less than 50 years old were infrequent and found in around $9.1 \%$ of mammograms. The prevalence of calcifications for the female population ranged from 9 to $17 \%$, while in women aged 65 years and above, the prevalence increased with age and exceeded 50\% [3].

Cardiovascular disease and breast cancer were two of the main causes of mortality in women. Mammography was recommended for all women from the age of 40 to 50 years as a screening test for breast cancer. Mammographically detected breast arterial calcifications which discovered as incidentally finding were considered without clinical importance, as they were not accompanied by increased risk of breast cancer [4].

However, the relation between breast arterial calcifications on mammograms and the cardiovascular risk 
factors as well as the atherosclerotic cardiovascular disease had been shown in recent studies. The results of these studies suggested that breast arterial calcifications, which were detected during routine mammography, could be valuable in identifying asymptomatic women at increased cardiovascular risk [5].

Mönckeberg medial calcific sclerosis (MCS) was a ring-like calcification of small- to medium-sized vessels without associated intimal thickening, appeared as "Railroad track" with classic radiographic linear pattern of calcifications on mammography [6].

\section{Correlation between BAC and heart disease}

According to Saxena et al. 2005, the pathogenesis of $\mathrm{MCS} / \mathrm{BAC}$ were related to several factors, including agerelated change, diabetes mellitus, chronic renal failure, and coronary artery disease [6].

\section{Risk factors}

I. Vascular calcification of the breast:

- Some studies had showed associations between vascular calcifications in breast tissue and chronic diseases such as diabetes, systemic arterial hypertension, coronary artery disease, kidney failure, autonomic neuropathy, and hypervitaminosis D [7].

- Arterial calcification in the breast could be associated with diabetes and hyperparathyroidism [8].

\section{Coronary calcification:}

(a) The major risk factors that could not be changed:

- Increasing age-Over $83 \%$ of people who died of coronary heart disease were 65 or older.

- Male sex (gender)-Men showed a greater risk of heart attack than women do, which tended to occur earlier in life.

- Heredity (including Race)—Children of parents with heart disease were more likely to have heart diseases [9].

(b) The major risk factors you could modify, treat, or control by changing your lifestyle or taking medicine:

- Tobacco smoke-Smokers' risk was 2-4 times of developing coronary heart disease than that of nonsmokers.
- High blood cholesterol-As blood cholesterol rised, the risk of coronary heart disease increased.

- High blood pressure-High blood pressure that increased the heart's workload could cause the heart to thicken and become stiffer.

- Physical inactivity.

- Obesity and overweight-People who have exceed body fat especially at the waist were likely to have heart disease and stroke even if there was no other risk factors.

- Diabetes mellitus [9].

\section{Methods}

In our study, screening mammography was performed in 100 women, whose ages ranged from 40 to 80 years with the mean age 56.8 years, who had been examined with different cardiac investigations for the evaluation of coronary cardiac disease/risk. The presence of $\mathrm{BAC}$, number of affected vessels, and the distribution of calcification in the vessel wall were evaluated in the mammography. Subjects were questioned in terms of the cardiovascular risk factors including diabetes mellitus, hypertension, and dyslipidemia. The cardiac investigations included cardiac catheterization, CT coronary angiography, and thallium scan.

\section{Inclusion criteria}

Our study included female patients with age ranging from 40 to 80 years who had been examined with cardiac investigations (cardiac catheterization, CT coronary angiography, or thallium scan) with either positive or negative results for coronary cardiac disease/risk.

\section{Exclusion criteria}

Not present.

\section{Possible risk}

No possible risk.

\section{Methodology}

All patients were submitted to the following:

- Full cardiac history (presence of risk factors and past history of cardiac ischemic insults)

- Cardiac investigations including one of the following cardiac catheterization (either diagnostic or therapeutic), CT coronary angiography, or thallium scan. All the previous cardiac imaging were used to evaluate coronary artery calcifications, vascular calcium deposition and scoring, atherosclerotic arterial changes and narrowing.

- Bilateral digital mammography as part of the annual screening program was performed in mediolateral oblique and craniocaudal views using the digital mammography system (Senographe 2000 D, GE). 
The presence of BAC, number of affected vessels, and the distribution of calcification in the vessel wall were evaluated in the mammography.

- Correlation between the mammographic findings and the cardiac investigation results.

\section{Results}

Our study included 100 female patients with their age ranging from 40 to 80 years old, with the mean age 56.8 years. They were subjected to cardiac investigations and mammography with evaluation of risk factors from their medical records. The results were as follows.

So our study showed that there was increased percentage of the BAC-positive cases among the 2nd group (54\%) which was the group with the higher age in comparison with the 1st group (17\%), showing an association between $\mathrm{BAC}$ and advancing age with a $p$ value $<0.001$.

There was increase in the mean age among the BACpositive cases of the 1st group in comparison with the BAC negative cases, while in the 2nd age group, the mean age was elevated in both BAC positive and negative cases.

Also there was increased percentage of cardiac cases in the 2nd age group in comparison with the 1st group with a significant $p$ value $=0.020$, showing an association between being a cardiac patient and advancing age (Figs. $1,2,3,4$, and 5).

And increased percentage of cardiac cases among the BAC-positive group in the 2nd group with a significant $p$ value $=0.022$ unlike the 1 st group which showed no correlation.

In the 1st group, there was decreased percentage of risk factors positive among the BAC-positive group, while in the 2nd group, there is increase in the risk factors positive cases in both positive and negative BAC groups, meaning there was no correlation between presence of BAC and having risk factors in either age groups (Tables 1, 2, 3, and 4).

\section{Discussion}

Breast arterial calcifications which were detected during routine annual mammography screening were considered an incidental finding as they were not associated with increased risk of breast cancer. The goal of this research was to find a correlation between the presence of BAC on mammography and cardiovascular disease and their risk factors. Thus, BAC detected during routine mammography could be valuable in identifying asymptomatic women at increased future CVD risk that may be candidates for more aggressive management.

According to Ugur Topal 2007, trying to prove the relationship between BAC and coronary artery disease those were his results: eighty (65\%) of 123 patients had CAD. BAC was found in the mammography of 49 (39.8\%) patients. The duration and ages of menopause of the patients with BAC were significantly higher than those without $\mathrm{BAC}(p<0.001)$. And there was also significant correlation between the BAC and Gensini scores $(p=0.059)$. As well there was an increase in the frequency of BAC among patients with more than two vessels with stenosis $(p=0.033)$ [10], compared with our study, there was a significant correlation between BAC and CAD $(p=0.006)$, and the mean age of patients with BAC was 63.7 in comparison with the mean age for those with no BAC was $53.9(p=0.000)$.

According to Paul S. Dale 2008, which was also trying to prove the ability of BAC detected on screening mammography to discover women with increased risk of CAD and diabetes showed the following results: of 1000 consecutive women undergoing screening mammography,

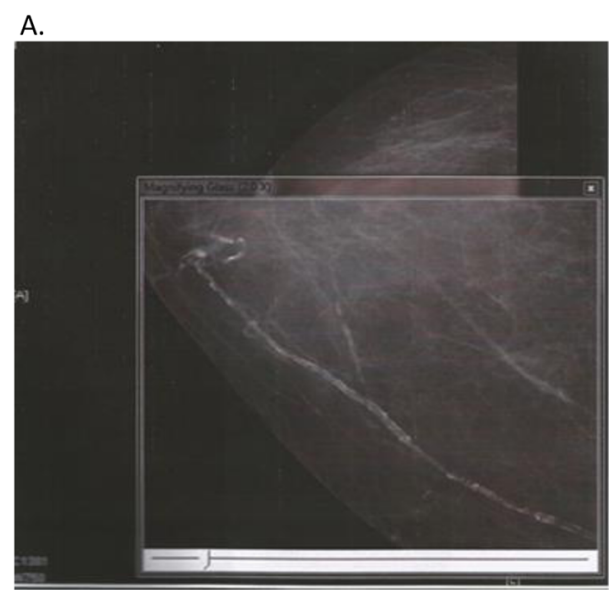

B.

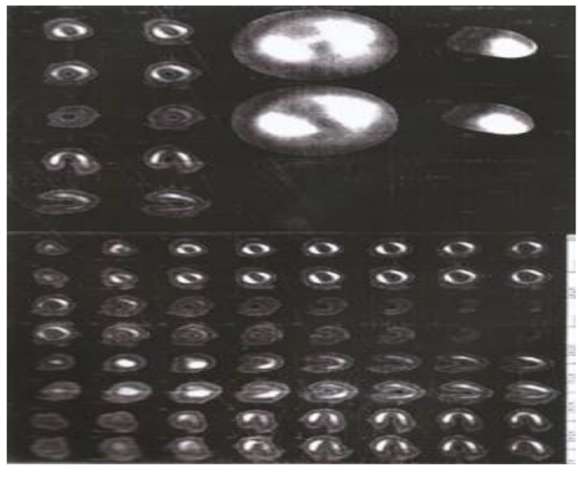

Fig. 1 a 58-year-old female patient with chest pain on rest and exertion. Her radioactive isotope scan showed ischemia and small scar at the LAD territory and mild ischemia at the RCA territories. CC mammography view showing unilateral BAC. b Showing thallium scan of the previously mentioned lesions 


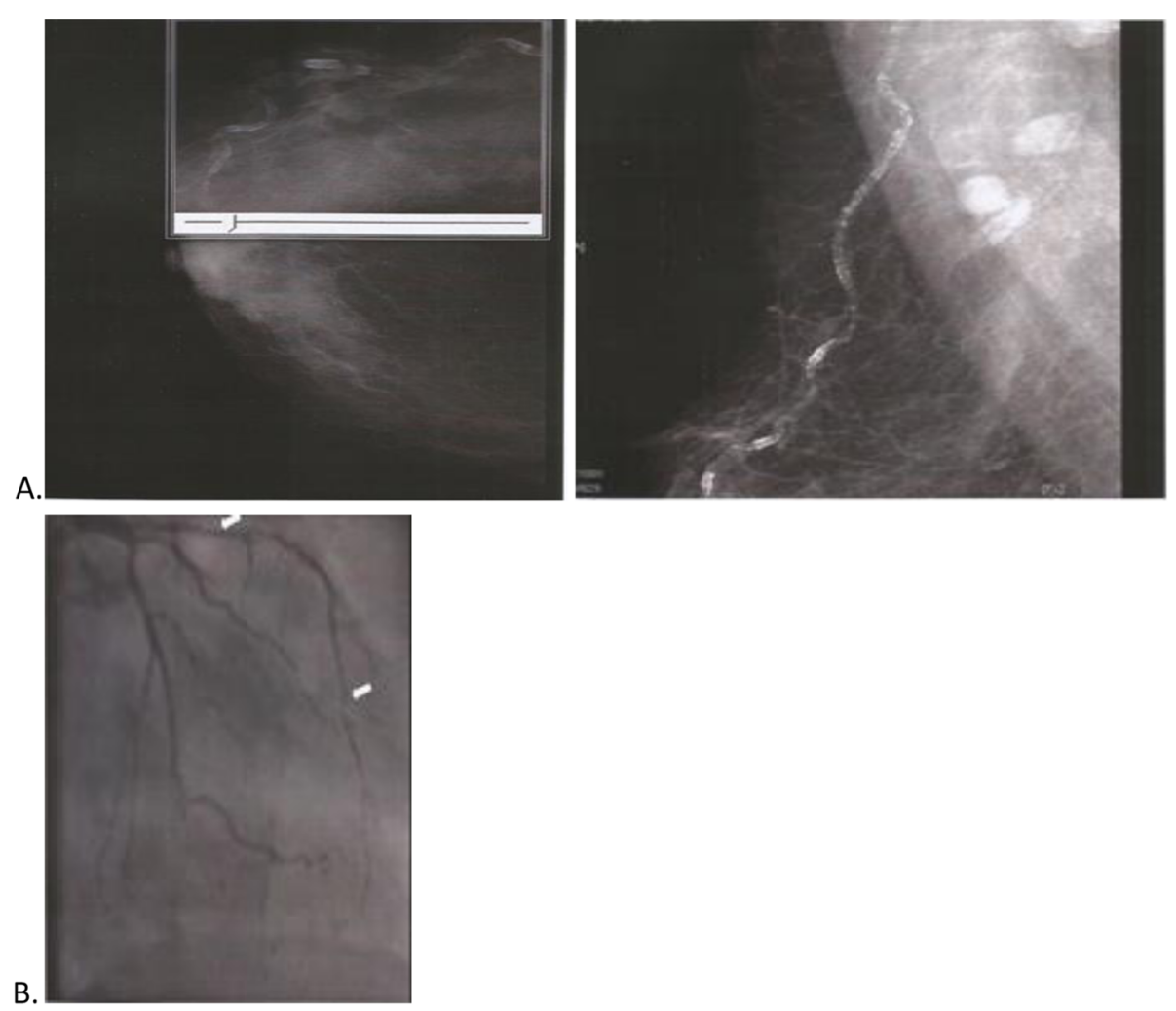

Fig. 2 a 68-year-old female patient known HTN, dyslipidemia, and chest pain on exertion, patient's CT coronary angiography revealed $90 \%$ stenosis of the proximal and mid part of left anterior descending artery. CC and MLO mammography images showing unilateral BAC. $\mathbf{b}$ Stenosis of LAD as seen in cardiac catheterization

181 showed a history of CAD and/or diabetes. Of those women without diabetes or heart disease, 86 of 819 (10.5\%) had BAC. One hundred forty women had diabetes, of whom 57 (40.7\%) had BAC, and 72 women had CAD, and of whom 36 (50\%) had BAC. The association among $\mathrm{BAC}, \mathrm{CAD}$, and diabetes was extremely significant $(p<.0001,95 \%$ confidence interval $[\mathrm{CI}])$. The odds ratio of having diabetes or CAD with BAC was 4.3 and 3.6 times greater than the odds of having these diseases without BAC. This large prospective study showed a significant association between BAC detected on screening mammography and if there was a personal history of CAD and diabetes, detecting that screening mammography may identify women at increased risk for these diseases [11], compared with our study, the association between BAC and CAD showed an odds ratio of 2.9 and (CI 0.96-9.218)

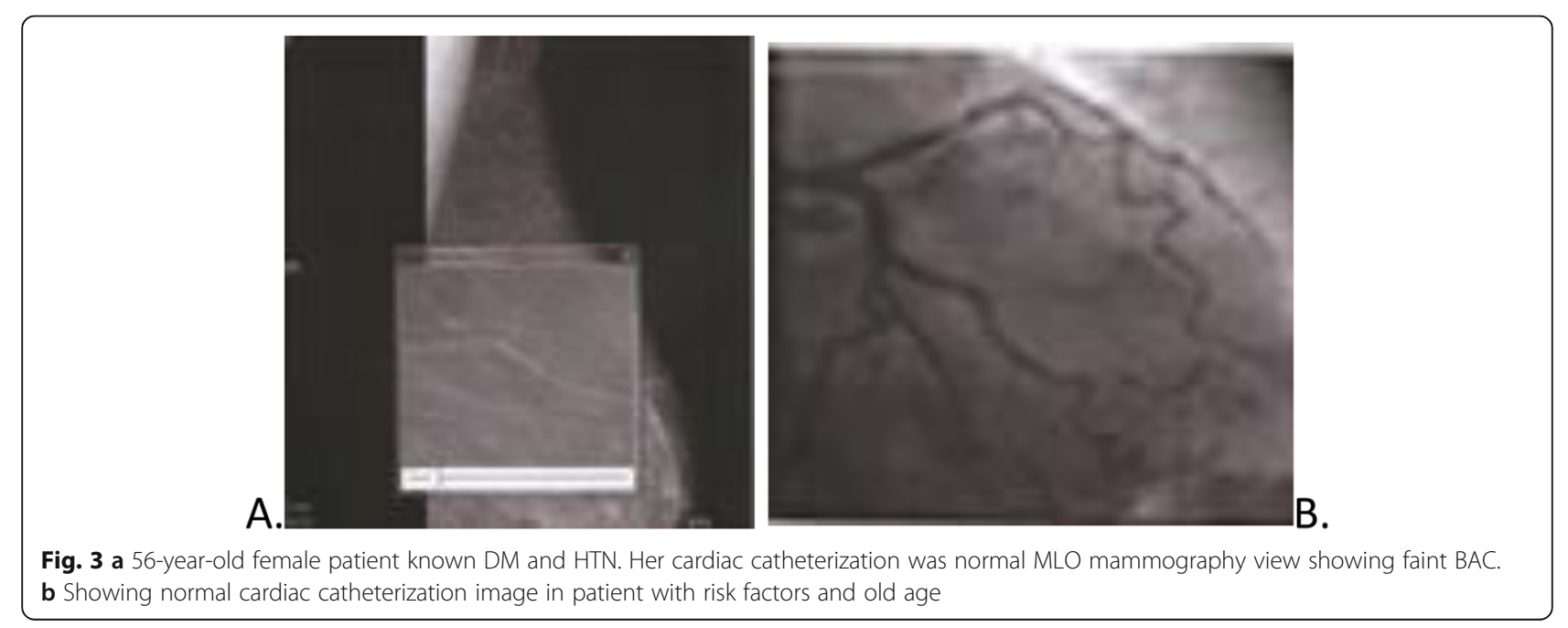



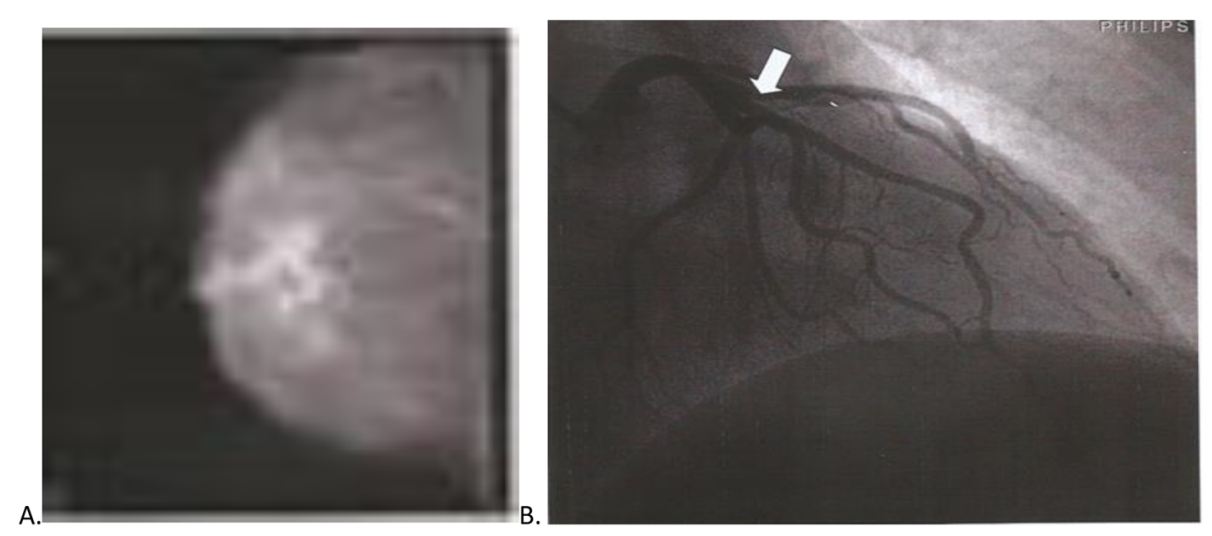

Fig. 4 49-year-old female patient known DM and chest pain on exertion and rest, cardiac catheterization revealed stenotic lesions at the proximal segment of left anterior descending artery. Bilateral breast mammography showing no BAC. $\mathbf{b}$ This is an example of CAD patient with no evidence of BAC

while no association between BAC and risk factors (DM, HTN, dyslipidemia) showing the following $p$ value 0.47 .

According to Evelling L. C. Oliveira 2009, a casecontrol study consisting of 40 women with coronary artery disease in the case group and 40 women without any history of coronary artery disease in the control group both were matched according to age and aiming to prove that BAC detected on mammography as an independent factor indicating coronary artery disease [12].

Forty women with coronary artery disease, who were included in the case group, underwent mammography during the preceding 12 months. Cases which were proven as coronary artery disease cases either confirmed by coronary angiography or had experienced at least one episode of acute myocardial infarction [12].

Vascular calcification in breast tissue was defined as the detection of parallel linear calcified tracks along the course of a vessel that was detected on at least one mammographic viewing plane. The intensity of the calcifications, number of vessels affected and the side affected were all analyzed. The presence and intensity of the calcifications were graded as follows: Absent: No vascular calcifications; slight: Arteries that showed little calcification with distances greater than $10 \mathrm{~mm}$ between calcified areas, moderate: Arteries obviously outlined by calcifications over a considerable proportion of their course and severe: Arteries extensively affected, had almost continuous columns of calcification, with at least two branches were visible. All mammograms were interpreted by only one observer. The number of calcified vessels was detected independently of the intensity of calcification. The laterality of the calcification was also detected and graded as unilateral, bilateral [12].

The odds ratio (OR) for coronary artery disease was 3.56 , in relation to vascular calcifications in breast tissue seen via mammography, compared with our study, it was 2.97 .

In Evelling study [12], the odds ratios for the other risk factors analyzed were 4.20 for diabetes mellitus, 12.33 for arterial hypertension, and 2.53 for a family history of coronary artery disease, in agreement to our study which found no association between BAC and risk factors

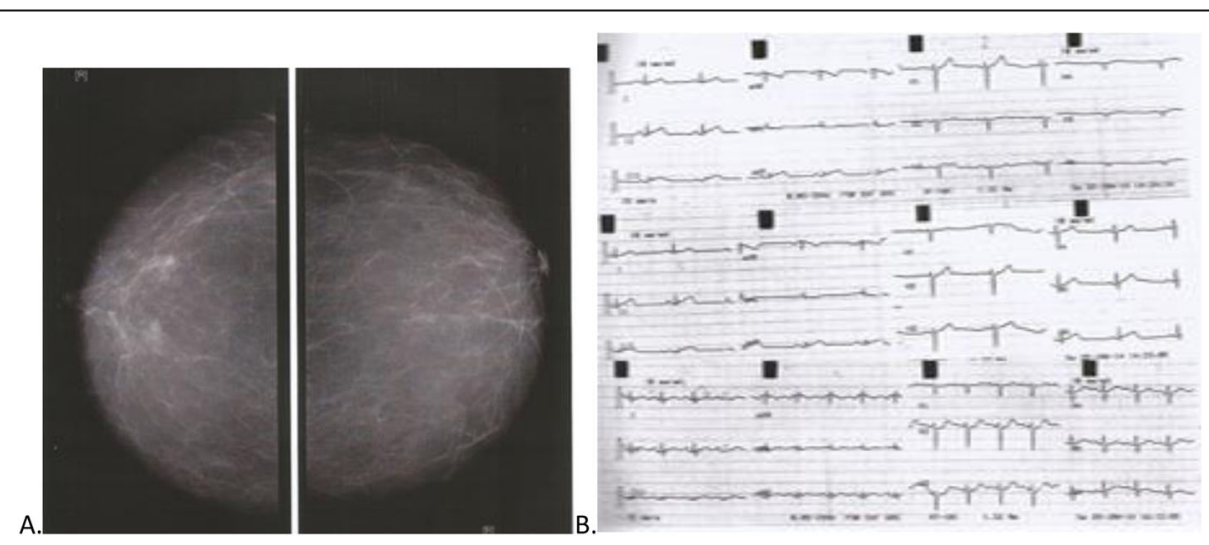

Fig. 5 51-year-old female patient known DM, diagnosed with inferior STEMI by ECG, cardiac enzymes and echocardiogram. Bilateral breast mammography CC view showed no evidence of BAC. $\mathbf{b}$ ECG of an inferior STEMI. Example of CAD patient without BAC 
Table 1 Data description of the cases

\begin{tabular}{lll}
\hline & & Count \\
\hline BAC & Negative & 71 \\
& Positive & 29 \\
CAD & Negative & 59 \\
& Positive & 41 \\
Risk factors & Negative & 47 \\
& Positive & 53 \\
Group & 40-60 years & 69 \\
& 61-80 years & 31 \\
\hline
\end{tabular}

The 100 patients were divided into two groups according to age: 1st group, age ranging from 40 to 60 years; 2 nd group, age ranging from 61 to 80 years

(DM, HTN, dyslipidemia) in both 1st and 2nd age groups showing the following $p$ values respectively 0.56 and 0.24 .

According to Evelling L.C.Oliveira 2009 [12], vascular calcifications detected in breast tissue via mammography were usually bilateral. A statistically significant difference in the intensity of the calcifications between the two groups was noticed. The median number of calcified vessels in the case group was three and none in the control group $(p<0.01)$.

In their analysis, the presence of vascular calcifications in breast tissue seen via mammography was an independent risk factor for coronary artery disease as they found. Some previous studies had also proven such an association. In previous studies, vascular calcifications seen via mammography were noticed in 16 to $31 \%$ of women with coronary artery disease [12].

In a study of 131 women in 2007, Ferreira et al. showed an odds ratio of 2.96 (CI 1.25-7.30) [13], compared with our study, I found an OR 2.97 (CI 0.9609.218).

One of the interesting findings was that when the vascular calcifications in breast tissue were bilateral, the atherosclerotic process and consequent vascular calcification were almost affecting the entire arterial system [12].

According to Mohammad H. Zgheib 2010, this study showed the following results: Fifty-seven patients (mean age, 72 years 69.8 [standard deviation]) had BAC-

Table 2 Comparison between the two groups

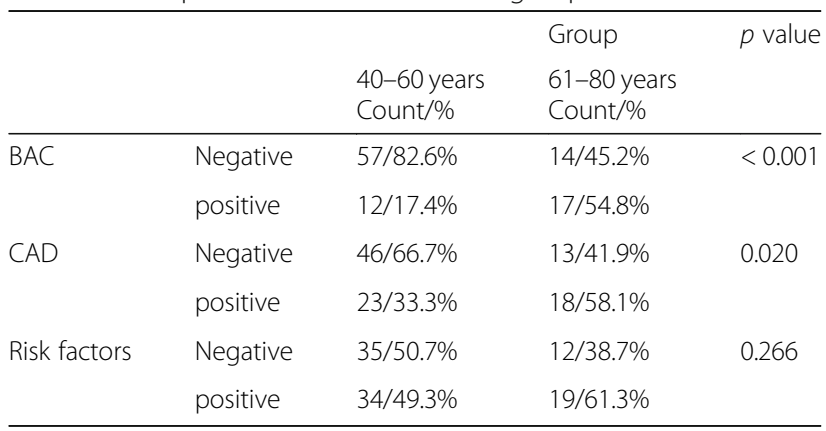

positive lesions. The 115 patients (mean age, 60.4 years 6 11) without BAC were younger than those with BAC $(p<.001)$ and BAC was significantly associated with some cardiac risk factors. Approximately $61 \%$ of patients with BAC had a family history of CHD, compared with only $44 \%$ of patients without BAC $(p=.034)$. Presence of BAC was significantly accompanied by history of CHD (odds ratio, 2.66; 95\% CI, 1.31, 5.42), CHD-equivalent disease (peripheral vascular disease, transient ischemic attack, stroke, or angina) (odds ratio, 2.25; 95\% CI, 1.05, 4.79), and family history of CHD (odds ratio, 2.08; 95\% CI, 1.08, 3.98). BAC showed no significant association with smoking, postmenopausal status, or hyper lipidemia and on the other hand, it had an association with hypertension $(p=.051)$ and diabetes $(p=.097)$.

At coronary angiography, some evidence of CHD (grades 1-4) were found in 104 patients and 68 had completely normal findings. The prevalence of BAC in patients with CHD was $36 \%$ versus $29 \%$ of patients without CHD $(p=.40)$. We found out no correlation between BAC and any degree of CHD or distribution of stenosis. Of the 83 patients with advanced CHD (70\% or greater stenosis, grade 4 ), only $41 \%$ patients had BAC versus $59 \%$ of patients who had not $(p=.14)$ [14], while according to our study, the 1st group of patients (with higher age) showed that $76.5 \%$ of the BAC-positive cases of this group were cardiac patients showing a significant $p$ value 0.022 , meaning a correlation between BAC positive and being cardiac in 2nd group patients only. There was no association between BAC and risk factors in 1st and 2nd age groups with insignificant $p$ values $=0.56$ and 0.24 respectively.

According to Hekimoğlu et al. 2012, who was aiming to discuss intramammarian arterial calcifications value in the prediction of coronary artery disease, mammography was done on 55 women over 40 years of age who do coronary angiography and did not have a mammography in the past year. Coronary angiography results, intramammarian arterial calcifications, and coronary artery disease risk factors were all evaluated. The percentage of intramammarian arterial calcifications was $41.8 \%$. A significant relationship between coronary artery disease and intramammarian arterial calcifications was proven (OR 10.8, 95\% Cl 3.02-38.59). The negative predictive value and positive predictive value of intramammarian arterial calcifications for coronary artery disease were $75 \%$ and $78.3 \%$ respectively. Also advancing age was detected to be relevant with these calcifications (OR $1.15,95 \% \mathrm{Cl} 1.05-1.25)$. The idea that the mammography was in use as a screening tool among women over 40 and could be used also in coronary artery disease risk assessment was supported by the results in the present literature which should be confirmed by further larger group controlled studies [15], compared with our study 
Table 3 Relation of BAC in 1st group (40 to 60 years) with age, cardiac conditions, and risk factors

\begin{tabular}{|c|c|c|c|}
\hline $\begin{array}{l}\text { 1st group: age range from } 40 \text { to } 60 \text { years } \\
69 \text { cases }\end{array}$ & $\begin{array}{l}\text { BAC positive } \\
\text { Count/\% }\end{array}$ & $\begin{array}{l}\text { BAC negative } \\
\text { Count } / \%\end{array}$ & $p$ value \\
\hline \multicolumn{4}{|l|}{ CAD } \\
\hline Positive & $5 / 41.7 \%$ & $18 / 31.6 \%$ & \multirow[t]{2}{*}{0.517} \\
\hline Negative & $7 / 58.3 \%$ & $39 / 68.4 \%$ & \\
\hline \multicolumn{4}{|l|}{ Risk factors } \\
\hline Positive & $5 / 41.7 \%$ & $29 / 50.9 \%$ & \multirow[t]{2}{*}{0.562} \\
\hline Negative & $7 / 58.3 \%$ & $28 / 49.1 \%$ & \\
\hline \multirow[t]{4}{*}{ Age } & \multicolumn{2}{|l|}{ BAC } & \multirow[t]{3}{*}{$p$ value } \\
\hline & \multicolumn{2}{|c|}{ Positive/negative } & \\
\hline & \multicolumn{2}{|c|}{ Mean-SD-Min-max/mean-SD-Min-max } & \\
\hline & \multicolumn{2}{|c|}{$\begin{array}{l}56.92-4.29-45.00-60.00 \\
50.68-5.09-40.00-60.00\end{array}$} & $<0.001$ \\
\hline
\end{tabular}

which showed a correlation between BAC and CAD in the 2nd group only with a significant $p$ value $=0.022$ less, there was an increase in the mean age among BACpositive cases in both age groups but it was more significant in 1 st group with a $p$ value $<0.001$ while in the 2 nd group, the mean age in BAC-positive cases was 68.5 and in BAC-negative cases was 67.3 giving an insignificant $p$ value 0.605 . There was no significant association between BAC and risk factors in both age groups with insignificant $p$ values.

According to Maryam Moradi 2014, the results of this study which was done to evaluate the association between BAC and CTCA findings were as follows: the mean age of subjects without BAC $(n=115)$ was significantly lower than patients with BAC $(n=35)(68.03 \pm$ 6.16 versus $54.36 \pm 7.63$ years, $p<0.0001)$. However, women with BAC showed significantly high relative frequency of different grades of coronary artery stenosis $(p<0.0001)$, which was the same as our study in the 2nd age group [16].
According to Maryam Moradi 2014, although they showed higher prevalence of BAC in patients with more severe coronary artery stenosis, this could be related to the effects of age as a co-variant. This could mean that both $\mathrm{BAC}$ and the presence of more significant coronary artery stenosis might be independently correlated with age; and as a result, older women were more susceptible to have both BAC and coronary artery stenosis. In their study, the BAC-positive patients were significantly older than the other group. Further analyses had confirmed the role of age in the correlation of BAC and CTCA findings [16].

To sum up, from the six articles compared with our study, only one showed no correlation between BAC and $\mathrm{CAD}$ which was the same results as the 1st age group while the rest showed a significant correlation between BAC and CAD as the results of the 2nd age group. Therefore, detection of arterial calcifications on mammography should be reported and further correlation with age should be done so that in old patients

Table 4 Relation of BAC in 2nd group (61 to 80 years) with age, cardiac conditions, and risk factors

\begin{tabular}{lll}
\hline 2nd group: age range from 61 to 80 years 31 cases & BAC positive Count/\% & BAC negative Count/\% $\%$ \\
CAD & $13 / 76.5 \%$ & $5 / 35.7 \%$ \\
Positive & $4 / 23.5 \%$ & $9 / 64.3 \%$ \\
Negative & & $7 / 50.0 \%$ \\
Risk factors & $12 / 70.6 \%$ & $7 / 50.0 \%$ \\
Positive & $5 / 29.4 \%$ & BAC \\
Negative & BAC & Negative \\
Age & Positive & Mean-SD-min-max \\
& Mean-SD-min-max & $67.36-4.36-61.00-75.00$ \\
\hline
\end{tabular}


above 60 years, cardiac investigations have to be suggested.

\section{Conclusion}

Incidental detection of breast arterial calcification in mammography in females above 60 years warrants further evaluation of their coronary atherosclerotic state and risk of future development of serious coronary artery disease.

\section{Abbreviations}

BAC: Breast arterial calcifications; BIRADS: Breast imagimg and reporting data system; CAD: Computer-aided detection; CAD: Coronary artery disease: CC: Craniocaudal; CHD: Coronary heart disease; CTCA: CT coronary angiography; CVD: Cardiovascular disease

\section{Acknowledgements}

First and foremost, thanks to Allah, the most beneficial and most merciful. It is but for His mercy that we can put through in life.

\section{Authors' contributions}

AM analyzed and interpreted the patient data regarding the breast lesions. EA performed the ultrasound examination of the breast and was a major contributor in writing the manuscript. NA participated in writing the final manuscript. All authors read and approved the final manuscript.

\section{Funding}

Not applicable

\section{Availability of data and materials}

The data sets used and /or analyzed during the current study are available from the corresponding author on reasonable request.

\section{Ethics approval and consent to participate}

The study is a prospective study that was reviewed by the ethics committee of radiology departments and was approved by the review board that is related to our University Patients included gave informed written consent to use their data in research work. No applicable reference number.

\section{Consent for publication}

All the patients included in this research gave written informed consent to publish the data contained within this study.

\section{Competing interests}

The authors declare that they have no competing interests.

\section{Author details}

${ }^{1}$ Radiology Department (Women's Imaging Unit), Kasr El-Ainy Hospital ,Cairo University, Cairo, Egypt. ²Diagnostic and Intervention Radiology Department, Cairo University Hospitals, Kasr Al-Ainy, El-Manial, Cairo 11956, Egypt.

${ }^{3}$ Cardiology Department, Cairo University Hospitals, El-Manial, Cairo, Egypt.

Received: 29 November 2019 Accepted: 2 December 2019

Published online: 12 December 2019

\section{References}

1. Kataoka M, Warren R, Luben R et al (2006) Calcification of cardiovascular disease and risk factors when found at screening mammography? Am J Roentgenol 187(1):73-80

2. Iribarren C, Curr SM (2013) Breast arterial calcification: a new marker of cardiovascular risk? Cardiovasc Risk Rep 7(2):126-135

3. Akinola RA, Ogbera OA et al (2011) Mammograms and breast arterial calcifications: looking beyond breast cancer: a preliminary report. BMC Res Notes 4:2007

4. Crystal P, Zelingher J et al (2004) Breast arterial calcifications as a cardiovascular risk marker in women. Exp Rev Cardiovasc Ther 2(5):753-760

5. Rafeh NA, Castellanos MR et al (2012) Association between coronary artery disease diagnosed by coronary angiography and breast arterial calcifications on mammography: meta-analysis of the data. J Womens Health 21(10): 1053-1058

6. Saxena A, Waddell C et al (2005) Monckeberg medial calcific sclerosis mimicking malignant calcification pattern at mammography. J Clin Pathol 58(4):447-448

7. Evelling O, Freitas-Junior R et al (2009) Vascular calcifications seen on mammography an independent factor indicating coronary artery disease. Clinics 64(8):763-767

8. Stephens T (2009) Breast, benign calcifications. Clinics 64:8

9. Micha R, Wallace SK, Mozaffarian D (2010) Red and processed meat consumption and risk of incident coronary heart disease, stroke, and diabetes mellitus: a systematic review and meta-analysis. Circulation 121(21): 2271-2283

10. Topal U, Kaderli A et al (2007) Relationship between the arterial calcification detected in mammography and coronary artery disease. Eur J Radiol 63(3): 391-395

11. Dale PS, Richards M et al (2008) Vascular calcifications on screening mammography identify women with increased risk of coronary artery disease and diabetes. Am J Surg 196(4):537-540

12. Oliveira EL, Freitas-Junior R et al (2009) Vascular calcifications seen on mammography: an independent factor indicating coronary artery disease. Clinics (SaoPaulo) 64(8):763-767

13. Ferreira EMPF, Szejnfeld J et al (2007) Correlation between intramammary arterial calcifications and CAD. Acad Radiol 14:144-150

14. Zgheib MH, Buchbinder SS et al (2010) Breast arterial calcifications on mammograms do not predict coronary heart disease at coronary angiography. Radiology 254(2):367-373 RSNA 2010

15. Hekimoğlu B, Simşir BD et al (2012) The association of intramammarian arterial calcifications detected on mammography with coronary artery disease and its risk factors. J Belge de Radiologie 95(4):229-234

16. Maryam Moradi, Atoosa Adibi et al (2014). Relationship between breast arterial calcification on mammography with CT Calcium scoring and coronary $C T$ angiography results. Department of Radiology, Isfahan University of Medical Sciences, Isfahan. Advanced Biomedical Research. 3:79

\section{Publisher's Note}

Springer Nature remains neutral with regard to jurisdictional claims in published maps and institutional affiliations.

\section{Submit your manuscript to a SpringerOpen ${ }^{\circ}$ journal and benefit from:}

- Convenient online submission

- Rigorous peer review

- Open access: articles freely available online

- High visibility within the field

- Retaining the copyright to your article

Submit your next manuscript at $\boldsymbol{\nabla}$ springeropen.com 